\title{
Reperfusion Injury after Cranioplasty
}

\author{
Indranil Ghosh ${ }^{1}$ Suthirtha Hazra ${ }^{2}$ Amit Ghosh ${ }^{2}$ Bibhu Kalyani Das ${ }^{1}$
}

\author{
${ }^{1}$ Department of Neuroanaesthesiology, Institute of Neurosciences, \\ Kolkata, West Bengal, India \\ 2Department of Neurosurgery, Institute of Neurosciences, Kolkata, \\ West Bengal, India
}

J Neuroanaesthesiol Crit Care 2018;5:127-128.

Intracerebral hemorrhagic transformation of the gliotic area after cranioplasty is a very rare complication with only five reported cases to date. ${ }^{1-5}$ After obtaining informed written consent from the next of the kin, we present the case of a young male adult who suffered from bleeding involving the left cerebral hemisphere following cranioplasty.

A 30-year-old man presented at our hospital with past history of decompressive craniectomy done 6 months before due to left-sided acute subdural hematoma with left frontal contusion. On admission, he was conscious without any neurologic deficit. He was posted for elective autologous bone flap replacement. Preoperative computed tomographic (CT) scan showed craniectomy defect and gliosis at the site of previous site of injury ( - Fig. 1). He underwent cranioplasty under general anesthesia. Surgery was uneventful, and the dura was not breached. His postoperative

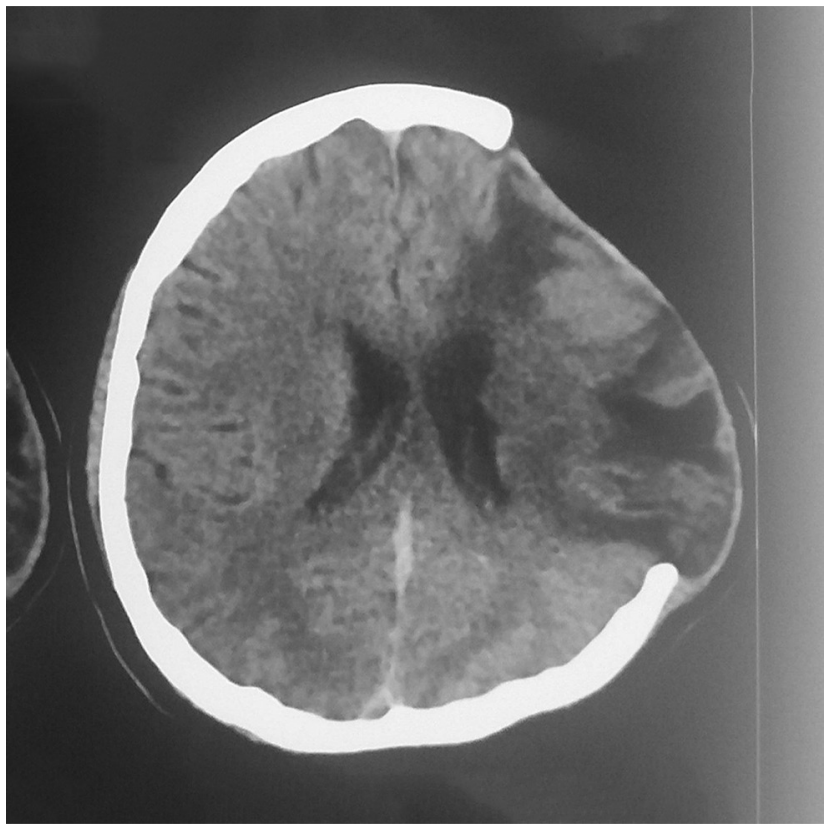

Fig. 1 Preoperative CT showing craniectomy defect and gliosis at the site of previous site of injury. CT, computed tomography
Address for correspondence Indranil Ghosh, MD, DNB, DM, Department of Neuroanaesthesiology, Institute of Neurosciences, "Sudha," Flat No. 4, 3rd Floor, 75/65 S. N. Roy Road, Kolkata 700017, West Bengal, India (e-mail: ghoshindranilghosh@rediffmail.com).

recovery was uneventful and shifted to the ward with an Aldrete score of 10 . Two hours postoperatively, the patient started becoming drowsy and developed right-sided weakness with slurring of speech. Immediate CT scan was done, which revealed hemorrhagic transformation of the previous gliotic area ( - Fig. 2). Considering the drop in Glasgow coma score (GCS) from 15 to 8, he was intubated and was taken to the operating theater for bone flap removal. Following the reexploration, he was ventilated overnight and extubated the next day. He was again conscious and obeying commands. Antiedema measures were continued for few more days along with antiepileptics. He was discharged in a conscious state with right-sided weakness and dysphasia. Transmission of atmospheric pressure decreases the volume of the brain and cerebrospinal fluid as the cranium is no longer a closed cavity after craniectomy. ${ }^{6}$ Improvement in

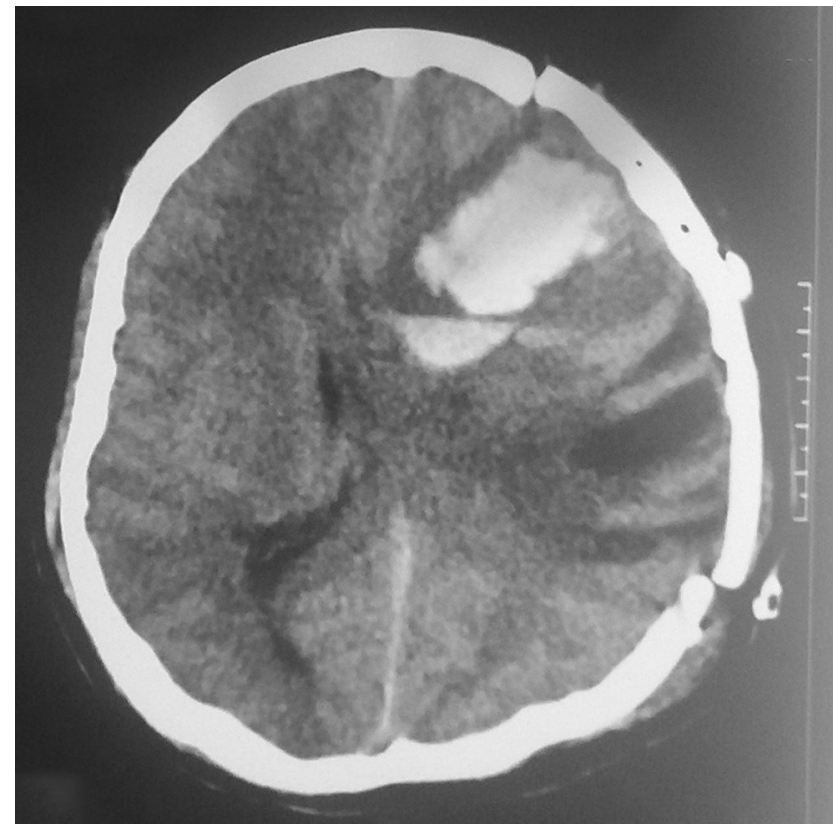

Fig. 2 Immediate CT showing hemorrhagic transformation of the previous gliotic area. $\mathrm{CT}$, computed tomography received

April 04, 2018

accepted

April 12, 2018

published online

May 18, 2018
DOI https://doi.org/

10.1055/s-0038-1654754.

ISSN 2348-0548.
Copyright (C2018 Indian Society of Neuroanaesthesiology and Critical Care
License terms

$($ (1) $\Theta \circledast$ 
cerebrovascular reserve capacity and glucose metabolism has been shown by transcranial Doppler and 18-fluorodeoxyglucose positron emission tomography. ${ }^{7}$ Yoshida et al reported significant increase in CBF (cerebral blood flow) and cerebral energy metabolism after cranioplasty by using ${ }^{133} \mathrm{Xenon} \mathrm{CT}$ and ${ }^{31} \mathrm{P}$ magnetic resonance spectroscopy. ${ }^{8} \mathrm{Also}$, increase in bilateral CBF has been demonstrated by ${ }^{\circ} \mathrm{A}$ perfusion studies. ${ }^{9}$ Hemorrhagic infarction has been reported as a complication of cranioplasty procedure. In our patient, hemorrhagic transformation in the gliotic area happened after cranioplasty, on the same side of operation. As there was no apparent injury either to a blood vessel or dura, mechanical cause of bleeding can be ruled out. Hence, our inference is that hemorrhagic transformation is related to the increased CBF to the operated side. Infra- and supratentorial hemorrhagic infarctions have been reported after craioplasty. ${ }^{2}$ By multiple neuroimaging, reperfusion, vessel injury, and venous stasis after cranioplasty have been held as possible mechanisms for such unique complication. ${ }^{3}$ In our patient since there was no infarction but only hemorrhage, we propose that rapid increase in bilateral CBF and volume in the chronic dysfunctional brain with questionable status of autoregulation probably resulted in venous stasis and congestion that may have increased the risk of hemorrhage. ${ }^{2}$ It is a rare complication of cranioplasty, but also of worth considering in patients who develop neurologic defect after cranioplasty.

\section{References}

1 Cecchi PC, Rizzo P, Campello M, Schwarz A. Haemorrhagic infarction after autologous cranioplasty in a patient with sinking flap syndrome. Acta Neurochir (Wien) 2008;150(4): 409-410, discussion 411

2 Chitale R, Tjoumakaris S, Gonzalez F, Dumont AS, Rosenwasser $\mathrm{RH}$, Jabbour P. Infratentorial and supratentorial strokes after a cranioplasty. Neurologist 2013;19(1):17-21

3 Eom KS, Kim DW, Kang SD. Bilateral diffuse intracerebral hemorrhagic infarction after cranioplasty with autologous bone graft. Clin Neurol Neurosurg 2010;112(4):336-340

4 Kwon SM, Cheong JH, Kim JM, Kim CH. Reperfusion injury after autologous cranioplasty in a patient with sinking skin flap syndrome. J Korean Neurosurg Soc 2012;51(2):117-119

5 Han JH, Lee MS. Intracerebral hemorrhagic infarction after cranioplasty in a patient with sinking skin flap syndrome. Korean J Neurotrauma 2016;12(2):152-155

6 Wang QP, Zhou ZM, You C. Paradoxical herniation caused by cerebrospinal fluid drainage after decompressive craniectomy. Neurol India 2014;62(1):79-80

7 Winkler PA, Stummer W, Linke R, Krishnan KG, Tatsch K. The influence of cranioplasty on postural blood flow regulation, cerebrovascular reserve capacity, and cerebral glucose metabolism. Neurosurg Focus 2000;8(1):e9

8 Yoshida K, Furuse M, Izawa A, Iizima N, Kuchiwaki H, Inao S. Dynamics of cerebral blood flow and metabolism in patients with cranioplasty as evaluated by $133 \mathrm{Xe}$ CT and 31P magnetic resonance spectroscopy. J Neurol Neurosurg Psychiatry 1996;61(2):166-171

9 Sakamoto S, Eguchi K, Kiura Y, Arita K, Kurisu K. CT perfusion imaging in the syndrome of the sinking skin flap before and after cranioplasty. Clin Neurol Neurosurg 2006;108(6):583-585 